\title{
DESIGNING PID CONTROLLER FOR DC MOTOR SYSTEM BY MEANS OF ENHANCED PSO ALGORITHM WITH DISCRETE CHAOTIC LOZI MAP
}

\author{
${ }^{1}$ Michal Pluhacek, ${ }^{1}$ Roman Senkerik, ${ }^{2}$ Donald Davendra, ${ }^{1}$ Ivan Zelinka \\ ${ }^{1}$ Tomas Bata University in Zlin, Faculty of Applied Informatics \\ Nam T.G. Masaryka 5555, 76001 Zlin, Czech Republic \\ \{pluhacek,senkerik,zelinka\}@fai.utb.cz
}

${ }^{2}$ Department of Computer Science, Faculty of Electrical Engineering and Computer Science
VB-TUO, 17.listopadu 15, 70833 Ostrava-Poruba, Czech Republic

\section{KEYWORDS}

PID controller, Optimization, Evolutionary Algorithms, PSO algorithm, Chaos, Lozi map.

\begin{abstract}
The main aim of this paper is the utilization of discrete chaotic Lozi map based chaos number generator to enhance the performance of PSO algorithm. This paper presents the results of research, in whichchaos enhanced PSO algorithm is used to design an optimal PID controller for DC motor system. Obtained results are compared with other non-heuristic and heuristic methods.
\end{abstract}

\section{INTRODUCTION}

Optimization started to play a crucial partforalmost every engineering and informatics tasks during recentyears. Optimization problems often representvery complex tasks and non-heuristic methods are very limited in finding of the proper solutions. As the complexity of optimization problems increases, thenonheuristic methods may not be able to solve them even in very distant future, whereasthe new heuristic methods can solve such tasks.Among these so called "softcomputing" methods belongevolutionary algorithms, which are inspired by evolution theory and natural behavior,and have helped to achieve very impressive results in solving various problems.

Recent study (Davendra et al., 2010) shows that chaos driven evolutionary algorithms might be very promising field of researchwithin evolutionary computation. The main principle is the utilization of chaos based number generator instead of a classic inbuilt computer randomness, which implementation may wary depending on used platform.

\section{Particle swarm optimization algorithm}

PSO (Particle swarm optimization algorithm)is the evolutionary optimization algorithm based on the natural behaviorof bird and fish swarms and was firstly introduced by R. EBERHART and J. KENNEDY in 1995 (Kennedy, Eberhart 1995, Eberhart,
Kennedy2001). As an alternative to genetic algorithms (Goldbeg, David, 1989) and differential evolution (Storn, Price, 1997), PSO proved itself to be able to find better solutions for many optimization problems. Term "swarm intelligence" (Eberhart,Kennedy, 2001) refers to the capability of particle swarms to exhibit surprising intelligent behavior assuming that some form of communication (even very primitive) can occur among the swarm particles (individuals).

In each generation, a new location of a particle is calculated based on its previous location and velocity, where by velocity is understood "velocity vector" i.e. velocity for each dimension of the problem.

One of the disadvantages of basic PSO algorithm is the rapid acceleration of particles which causes them toabandon the defined area of interest. For this reason, several modifications of PSO were introduced to handle with this problem.

Within this research, chaos driven PSO strategy with inertia weight was used. Default values of all PSO parameters were chosen according to the recommendations given in (Kennedy, Eberhart 1995, Eberhart, Kennedy 2001). Inertia weight is designed to influence the velocity of each particle differently over the time (Nickabadi et al., 2011). In the beginning of the optimization process, the influence of inertia weight factor $w$ is minimal. As the optimization continues, the value of wis decreasing, thus the velocity of each particle is decreasing, since $w$ is the number $<1$ and it multiplies previous velocity of particle in the process of new velocity value calculation. Inertia weight modification PSO strategy has two control parameters $w_{\text {start }}$ and $w_{\text {end }}$. New $w$ for each generation is then given by Eq. 1, where $i$ stand for current generation number and $n$ for total number of generations.

$w=w_{\text {start }}-\frac{\left(\left(w_{\text {start }}-w_{\text {end }}\right) * i\right)}{n}$

Chaos driven number generator is used in the main PSO formula (Eq. 2) that determines new "velocity"and thus the position of each particle in next generation (or migration cycle). 
$v(t+1)=v(t)+c_{1} \cdot$ Rand $\cdot(p$ Best $-x(t))+c_{2}$.

Rand $\cdot(g B e s t-x(t))$

Where:

$v(t+1)-$ New velocity of particle.

$v(t)$ - Current velocity of particle.

$\mathrm{c} 1, \mathrm{c} 2$ - Priority factors.

pBest - Best solution found by particle.

gBest - Best solution found in population.

$x(t)-$ Current position of particle.

Rand - Random number, interval $<0,1>$. Within Chaos

PSO basic inbuilt computer (simulation software) random generator is replaced with chaotic generator (in this case by using of Lozi map).

New position of particle is then given by Eq. 3, where $\mathrm{x}(\mathrm{t}+1)$ is the new position:

$x(t+1)=x(t)+v(t+1)$

\section{Lozi map}

The Lozi map is a simple discrete two-dimensional chaotic map. The Lozi map is depicted in Figure 1. The map equations are given in Eq. 4 and 5.The parameters used in this work are: $a=1.7$ and $b=0.5$ as suggested in (Sprott 2003).

$$
\begin{aligned}
& X_{n+1}=1-a\left|X_{n}\right|+b Y_{n} \\
& Y_{n+1}=X_{n}
\end{aligned}
$$

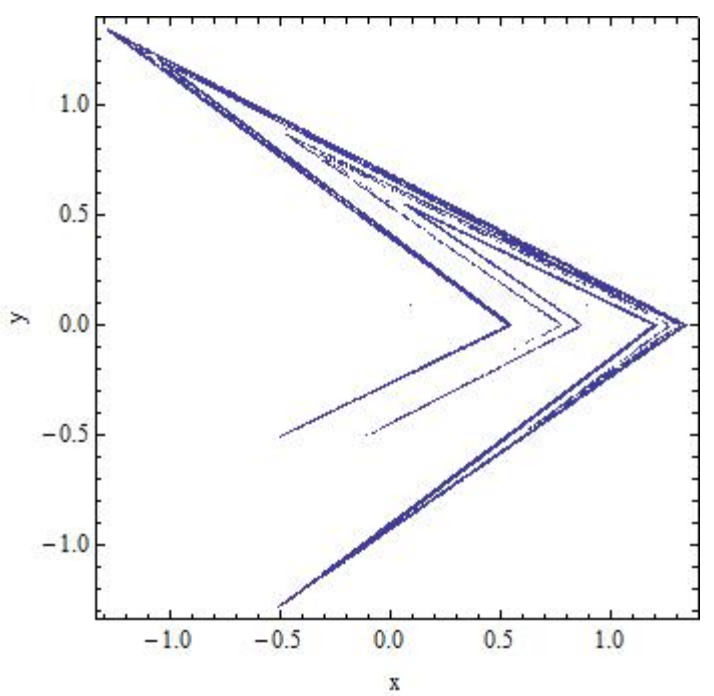

Figure 1: Lozi map

\section{PROBLEM DESIGN}

The PID controller contains three unique parts; proportional, integral and derivative controller (Astrom 2002). A simplified form in Laplace domain is given in Eq 6.
$G(s)=K\left(1+\frac{1}{s T_{i}}+s T_{d}\right)$

The PID form most suitable for analytical calculations is given in Eq7.

$G(s)=k_{p}+\frac{k_{i}}{s}+k_{d} s$

The parameters are related to the standard form through: $k_{p}=K, k_{i}=K / T_{i}$ and $k_{d}=K T_{d}$. Acquisition of the combination of these three parameters that gives the lowest value of the test criterions was the objective of this research.

The transfer function of used DC motor is given by Eq. 8. $[6,7]$

$$
G(s)=\frac{0.9}{0.00105 s^{3}+0.2104 s^{2}+0.8913 s}
$$

\section{Cost function}

Test criterion measures properties of output transfer function and can indicate quality of regulation. Following four different integral criterions were used for the test and comparison purposes: IAE (Integral Absolute Error), ITAE (Integral Time Absolute Error), ISE (Integral Square Error) and MSE (Mean Square Error). These test criterions (given by Eq. 9-12) were minimized within the cost functions for the enhanced PSO algorithm.

1. Integral of Time multiplied by Absolute Error (ITAE)

$$
I_{\text {ITAE }}=\int_{0}^{T} t|e(t)| d t
$$

2. Integral of Absolute Magnitude of the Error (IAE)

$$
I_{I A E}=\int_{0}^{T}|e(t)| d t
$$

3. Integral of the Square of the Error (ISE)

$$
I_{I S E}=\int_{0}^{T} e^{2}(t) d t
$$

4. Mean of the Square of the Error (MSE)

$$
I_{M S E}=\frac{1}{n} \sum_{i=1}^{n}(e(t))^{2}
$$

For further details see (Nagraj et al., 2008, Davendra et al., 2010).

\section{RESULTS}

All experiments were focused on the optimization of the four different specification functions as given in previous section. The best results of the optimization with corresponding values of $\mathrm{k}_{\mathrm{p}}, \mathrm{k}_{\mathrm{i}}$ and $\mathrm{k}_{\mathrm{d}}$ together with 
selected response profile parameters are presented in Table 1.

When tuning a PID controller, generally the aim is to match some preconceived 'ideal' response profile for the closed loop system. The following response profiles are typical (Landau, 2006):

Overshoot: this is the magnitude by which the controlled 'variable swings' past the setpoint. 5 - 10\% overshoot is normally acceptable for most loops.
Rise time: the time it takes for the process output to achieve the new desired value. One- third the dominant process time constant would be typical.

Settling time: the time it takes for the process output to die between, say $+/-5 \%$ of setpoint.

From the statistical reasons, optimization for each criterion was repeated 30 times. Results of the simple statistical comparison for the optimizations by means of chaos driven PSO algorithm are given in tables 2 and 3. Optimized system responses are depicted in Figures $2 \mathrm{a}-$ $2 \mathrm{~d}$ and compared in Figure 3.

Table 1: The best results for DC motor system

\begin{tabular}{|l|l|l|l|l|l|l|l|}
\hline Criterion & $\mathrm{CF}$ & $\mathrm{Kp}$ & $\mathrm{Ki}$ & $\mathrm{Kd}$ & Overshoot & Rise Time & Settling time \\
\hline IAE & 0.216732 & 247.484000 & 1.651430 & 58.458400 & 0.215909 & 0.010100 & 0.023300 \\
\hline ITAE & 0.006667 & 271.207000 & 0.050058 & 63.790800 & 0.233985 & 0.009500 & 0.031500 \\
\hline ISE & 0.018386 & 143.511000 & 30.512900 & 64.054000 & 0.223480 & 0.009500 & 0.032400 \\
\hline MSE & 0.000919 & 146.339000 & 28.788700 & 64.040300 & 0.223686 & 0.009500 & 0.032400 \\
\hline
\end{tabular}

Table 2: Average steady state responses for DC motor system

\begin{tabular}{|l|l|l|l|}
\hline Criterion & Avg. overshoot & Avg. rise time & Avg. settling time \\
\hline IAE & 0.221803 & 0.009917 & 0.025920 \\
\hline ITAE & 0.228235 & 0.009810 & 0.028243 \\
\hline ISE & 0.223643 & 0.009507 & 0.032380 \\
\hline MSE & 0.223739 & 0.009503 & 0.032387 \\
\hline
\end{tabular}

Table 3: Statistical overview of the criterion (CF) values for DC motor system

\begin{tabular}{|l|l|l|l|l|l|}
\hline Criterion & Max CF & Min CF & Avg. CF & Median & Std. dev. \\
\hline IAE & 0.307708 & 0.216732 & 0.234729 & 0.232278 & 0.017130022 \\
\hline ITAE & 0.045092 & 0.006667 & 0.018588 & 0.017136 & 0.008908 \\
\hline ISE & 0.018416 & 0.018386 & 0.018390 & 0.018386 & 0.000007 \\
\hline MSE & 0.000920 & 0.000919 & 0.000919 & 0.000919 & 0 \\
\hline
\end{tabular}

Results obtained for chaos PSO driven PID controller design are compared with previously published result (Nagraj et al., 2008) given by other heuristic and non-heuristic methods in table 4 .

Table 4: Comparison of other methods and proposed enhanced PSO

\begin{tabular}{|l|l|l|l|l|l|l|l|}
\hline Criterion & $\begin{array}{l}\text { Z-N (step } \\
\text { response) }\end{array}$ & $\begin{array}{l}\text { Kappa- } \\
\text { Tau }\end{array}$ & $\begin{array}{l}\text { Continuous } \\
\text { cycling }\end{array}$ & EP & GA & PSO & $\begin{array}{l}\text { PSO } \\
\text { Chaos }\end{array}$ \\
\hline IAE & 0.517600 & 0.518800 & 0.560000 & 0.489100 & 0.771200 & 0.916100 & 0.216732 \\
\hline ITAE & 3.380500 & 3.311300 & 7.820000 & 0.072100 & 0.378100 & 0.022900 & 0.006667 \\
\hline ISE & 2.346700 & 2.250300 & 3.200000 & 1.027700 & 1.043500 & 1.001600 & 0.018386 \\
\hline MSE & 0.011700 & 0.077778 & 0.016000 & 0.005100 & 0.005200 & 0.005000 & 0.000919 \\
\hline
\end{tabular}


Figure $2 a$
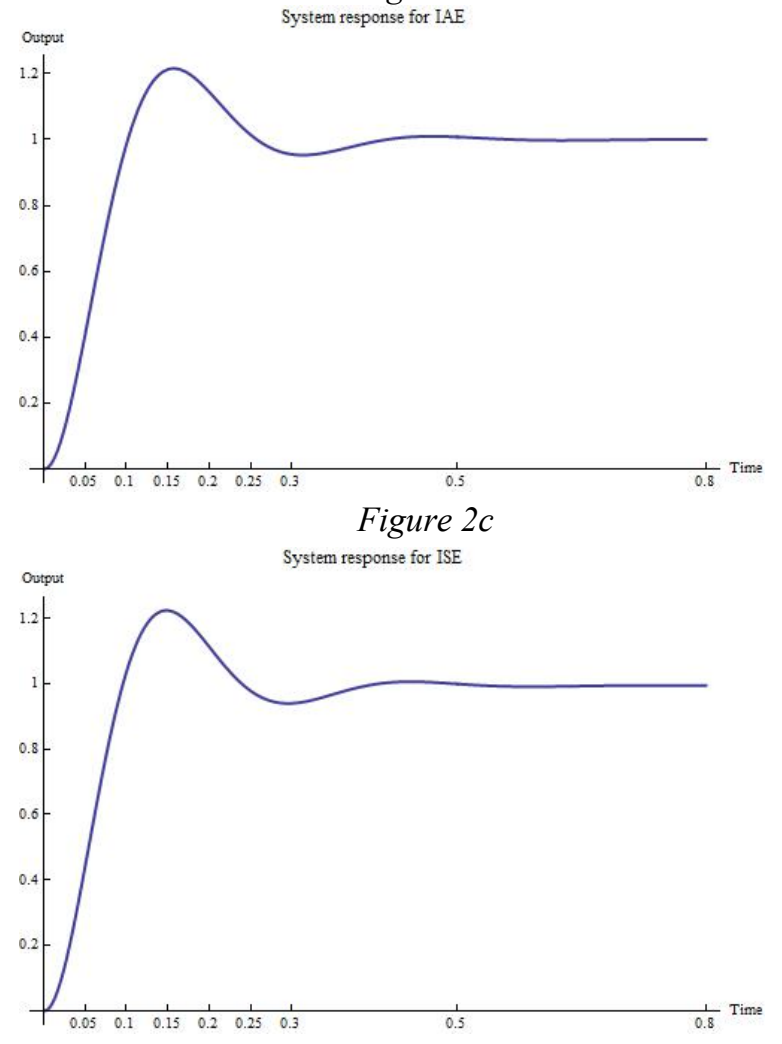

Figure $2 b$

system response for ITAE
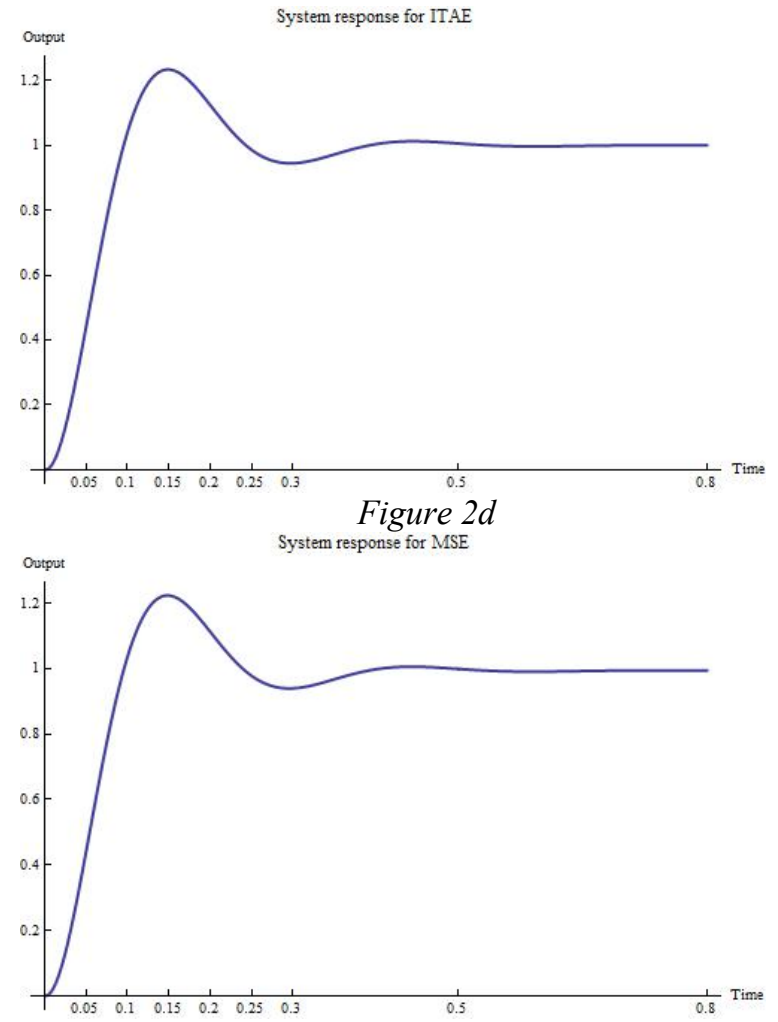

Figure 2: Optimized system responses for $\mathrm{K}_{\mathrm{p}}, \mathrm{K}_{\mathrm{i}}$ and $\mathrm{K}_{\mathrm{d}}$ obtained by four integral criterions.

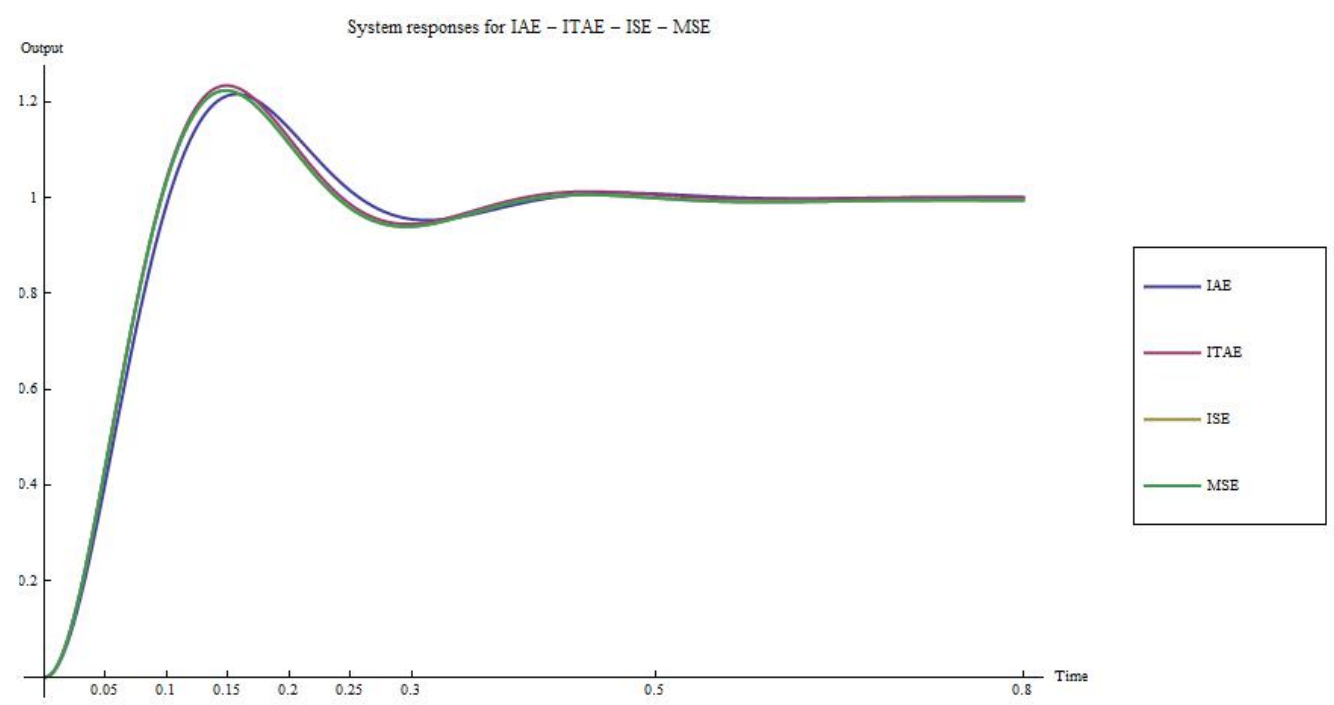

Figure 3: Comparison of optimized system responses for $\mathrm{K}_{\mathrm{p}}, \mathrm{K}_{\mathrm{i}}$ and $\mathrm{K}_{\mathrm{d}}$ obtained by four integral criterions.

\section{CONCLUSION}

This research was focused on the utilization of chaos driven PSO with discrete chaotic Lozi map for finding of the PID controller optimal settings for DC motor system.

Presented data and graphical simulation results lend weight to the argument that implementation of chaotic Lozi map as a random number generator into PSO algorithm may significantly improve its performance over other non-heuristic and heuristic methods in the case of solving the problem of optimal PID controller design for DC motor system.

Future research will be aimed to the possibilities of the development and improvement of the enhanced chaos driven PSO algorithm to achieve better results and explore more possible applications for this promising optimization approach. 


\section{ACKNOWLEDGEMENT}

This work was supported by European Regional Development Fund under the project CEBIA-Tech No. CZ.1.05/2.1.00/03.0089, and by Internal Grant Agency of Tomas Bata University under the project No. IGA/FAI/2012/037.

\section{REFERENCES}

Astrom K., “Control System Design”.Santa Barbra, California: University of California, 2002

Davendra D., Zelinka I., Senkerik R., "Chaos driven evolutionary algorithms for the task of PID control", Computers \& Mathematics with Applications, Volume 60, Issue 4, 2010, pp 1088-1104, ISSN 0898-1221.

Dorigo, M., Ant Colony Optimization and Swarm Intelligence, Springer, 2006.

Eberhart, R., Kennedy, J., Swarm Intelligence, The Morgan Kaufmann Series in Artificial Intelligence, Morgan Kaufmann, 2001.

\section{AUTHOR BIOGRAPHIES}

MICHAL PLUHACEK was born in the Czech

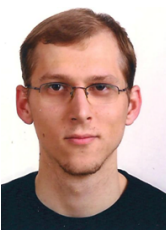
Republic, and went to the Tomas Bata University in Zlin, where he studied Information Technologiesand obtained his MSc degree in 2011. He is now a doctoral student at the same university. His email address is:pluhacek@fai.utb.cz

ROMAN SENKERIK was born in the Czech Republic,

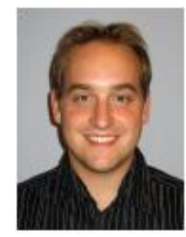
and went to the Tomas Bata University in Zlin, where he studied Technical Cybernetics and obtained his MSc degree in 2004 and Ph.D. degree in Technical Cybernetics in 2008. He is now a lecturer at the same university (Applied Informatics, Cryptology, Artificial Intelligence, Mathematical Informatics). His email address is: senkerik@fai.utb.cz

IVAN ZELINKA was born in the Czech Republic, and

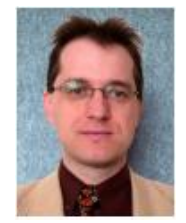
went to the Technical University of Brno, where he studied Technical Cybernetics and obtained his degree in 1995. He obtained Ph.D. degree in Technical Cybernetics in 2001 at Tomas Bata University in Zlin. Now he is aprofessor (Artificial Intelligence, Theory of Information).Email address: ivan.zelinka@vsb.cz .
Goldberg, David E. (1989). Genetic Algorithms in Search Optimization and Machine Learning. Addison Wesley. p. 41.ISBN 0201157675.

Kennedy, J.; Eberhart, R. (1995). "Particle Swarm Optimization".Proceedings of IEEE International Conference on Neural Networks. IV. pp. 1942-1948

Landau Y., "Digital Control Systems". Springer, London, 2006

Nagraj B., Subha S., and Rampriya B., "Tuning algorithms for pid controller using soft computing techniques", International Journal of Computer Science and Network Security, 2008. 8, pp.278-281.3

Nickabadi A., Mohammad Mehdi Ebadzadeh, Reza Safabakhsh, A novel particle swarm optimization algorithm with adaptive inertia weight, Applied Soft Computing, Volume 11, Issue 4, June 2011, Pages 36583670, ISSN 1568-4946

Sprott J. C., "Chaos and Time-Series Analysis", Oxford University Press, 2003

Storn R., Price K., Differential evolution - a simple and efficient heuristic for global optimization over continuous spaces, Journal of Global Optimization 11 (1997) 341359. 